\title{
Modeling Interactive Smart Spaces ${ }^{\star}$
}

\author{
Mattia Gianotti ${ }^{[0000-0001-6035-3367]}$, Fabiano Riccardi ${ }^{[0000-0001-5510-3240]}$, \\ $\underline{\text { Giulia Cosentino }}^{[0000-0002-8560-4924]}{ }^{\text {, Franca Garzotto }}{ }^{[0000-0003-4905-7166]}$, \\ and Maristella Matera ${ }^{[0000-0003-0552-8624]}$ \\ Department of Electronics, Information and Bioengineering, Politecnico di Milano, \\ Milano, Italy \\ \{name.surname\}@polimi.it
}

\begin{abstract}
The Internet of Things (IoT) enables the creation of Interactive Smart Spaces (ISSs) where different types of digital devices are integrated in the ambient or embedded in physical objects, and can sense human actions to control equipment, modify environmental parameters, or create multi-sensory effects. These IoT-enhanced interactive systems can support human activities in different contexts, e.g., education, entertainment, home assistance, rehabilitation, to name a few. This paper explores a human-centered perspective in the design of ISSs, which takes into account some salient characteristics of these systems and introduces new conceptual modeling issues going beyond representing hardware, software, and connectivity features of IoT devices. Interaction in particular emerges as a crucial modeling dimension, needed to capture the interplay occurring not only among multiple connected IoT devices but also among the users and the materials or the spaces embedding such devices. The paper presents a novel conceptual model for Interactive Smart Spaces and exemplifies the identified abstractions through a case study, modelling a complex ISS for children' play and learning that has been installed at two local schools and two therapeutic centers in our country.
\end{abstract}

Keywords: Interactive Smart Spaces · Interactive IoT $\cdot$ Smart Objects - Interaction modeling.

\section{Introduction}

In early '90s, Mark Weiser envisioned that pervasive devices and services would have become parts of our daily life [27]. Internet of Things (IoT) technologies have largely accelerated this trend. Initial IoT solutions focused on the creation of arrays of distributed connected sensors (Wireless Sensor Networks) to support automation systems relieving users from repetitive tasks (e.g., plants monitor or automatic controls). In this class of IoT systems, the interaction between the system and the user was of secondary priority. Nevertheless, thanks to the ever-increasing opportunity to integrate IoT into everyday objects and physical

\footnotetext{
* Supported by the Italian Ministry of University and Research (MIUR) under grant PRIN 2017 "EMPATHY".
} 
spaces, many environments we live in are augmented today through an interleaving between the cyber and the physical worlds [6]. Novel interactive systems are emerging that empower people in different activities and contexts of everyday life $[9,10,14,24]$. A more human-centered perspective has progressively emerged, in which the interactive capability of IoT-enhanced physical objects and spaces (hereinafter smart objects and smart spaces) becomes more and more central, raising new requirements and challenges for conceptual modelling. In this scenario, the scope of conceptual modeling would go beyond representing features related to hardware, software, connectivity, and communication among multiple devices, and should also address the interactions between the users and the materials or spaces embedding such devices. New conceptual modeling primitives are therefore needed to: i) represent the IoT-enabled interaction capabilities of smart objects and smart spaces, and their interactive behaviour, abstracting from the underlying technology; ii) describe how these features are orchestrated in order to offer an optimal smart interactive experience to the user [1]. Such primitives would help designers focus on the salient human-centered aspects of IoT-enhanced systems, paving the ground towards the definition of new methods and tools for interaction design in the IoT arena, as well as new technological architectures for this class of systems.

This paper presents an attempt to address this modelling challenge. After an overview of the relevant state of the art reported in Section 2, in Section 3 we discuss the relevant features that characterize Interactive Smart Spaces (ISSs) and classify them in order to contextualize our ISS model. Following the "separation of concerns" principle, we distinguish between two main design dimensions for ISSs, which results into two sub-models - Structural Model and Interactive Behavior Model, presented in Section 4. To exemplify our primitives and highlight their expressive power, Section 5 describes (fragments of) the conceptual model of a sophisticated multi-sensory ISS called "Magic Room". In Section 6 we depict the future directions of our research, and highlight the potential of an interaction-centered modeling approach for End-User Development research in the arena of interactive IoT systems.

\section{Related work}

In the last decades researchers had proposed different modelling approaches for IoT systems related to Smart Spaces. Four major topics emerge: privacy and security of data transmission $[2,25,26]$, orchestration of device behaviour $[4,12$, $21,23]$, data gathering and propagation $[8,19]$, and design of single devices and smart objects $[5,12]$. The role of the user is neglected or simply considered as a pure source of data, and existing approaches take into account only marginally (or not at all) the need of modeling human interaction in Interactive Smart Spaces. Haller et al. [18] present a distribution map for the computation and the interaction between what they call "augmented entities" and users through devices, resources and services without a distinction between human and nonhuman actors. Zeng et al. [28] describe the behaviour of a IoT-enhanced system 
through a two-levels Petri net. The top-level net describes the social flow between the different humans, and the physical flow between humans and objects; the lower level describes the flow of data between the digital twins of humans and the objects. Still, the model does not cover the interactive capabilities of smart physical objects. Gračanin et al. [17] present a two-tier architecture framework to encompass the interaction between the users and the system, but its expressiveness is limited to interactions that are directed by the user to the system through a client-based approach. In order to simulate and verify planned agents' distributed tasks, Zhao et al. [29] propose a methodology based on BIM-Sim $3 \mathrm{D}$ to simulate the human activities, their analysis and the configuration of the space. This method, however, may fail to capture the intrinsic unpredictability of users and describe the complex variability of activities. Markopoulos et al.[22] discuss how the Unified Modeling Language (UML) can be used as a reference representation language for interaction design; still, the authors do not provide any insight on how the UML primitives discussed can be applied to IoT-enhanced interactive systems.

\section{Characterizing Interactive Smart Spaces}

An Interactive Smart Space (ISS) is an ecosystem of smart resources, based on a variety of IoT devices [3], from basic sensors and actuators to digitally enhanced physical objects (smart objects [20]) and software services, featuring mutual cross-interactions and interactions with humans to support the accomplishment of user goals at multiple levels of complexity. ISSs call for a humancentered vision of IoT, where the involvement of human actors is central: it is the interactive dimension that distinguishes - at the highest degree - ISSs from other categories of "smart spaces" where the IoT technology is embedded in the physical environment and its physical components. Beside the high level of interactivity, another feature that characterizes many ISSs is the richness of digital media content and multisensory stimuli the user can experience in these environments. In a user-centered perspective, we can therefore characterize ISSs along two main dimensions:

- Interaction Intensity: interaction-intensive ISSs enable (and motivate) users to interact frequently and actively with the physical space and its components, using multiple modes of interacting with them, from touch and manipulation to gestures, movements, or voice, leading to a continuous exchange of actions and stimuli between the system and the user.

- Content Intensity: content-intensive ISSs provide a vast amount of digital media contents that are generated automatically, or by effect of interaction, offering engaging experiences enriched by multiple stimuli and, when contents are integrated in the fabric of the physical space, a feeling of immersiveness.

Different degrees of interaction intensity and content intensity lead to a wide spectrum of ISS types, ranging from Automated Interactive Smart Spaces (hav- 
ing low interaction intensity and low content intensity) to Multi-sensory Smart Spaces (having high interaction intensity and high content intensity).

An Automated Interactive Smart Space is a digitally-enriched physical space where the orchestration of IoT devices and the integrated software services are largely finalized to automating some tasks or ambient functionality. In many cases these ISSs focus on monitoring some variables characterizing the environment (e.g., temperature, luminosity, user presence or proximity) to enact actions that change the state of the physical environment by means of some physical devices installed in it (e.g., "switching on" the heating or the lights or "opening" the window blinds). In this class of systems, the interaction by human agents exists but it is often "implicit" or limited. For example, the user "implicitly" interacts with the room by entering it (which triggers the activation of the lighting). Explicit user actions are restricted to simple instructional tasks, such as controlling some devices or home appliances using a touch interface, manipulating a physical affordance, or issuing vocal commands to a conversational agents such as Alexa.

Multi-sensory smart spaces are characterized by providing multiple modes for the user to interact with devices, digital contents embedded in the physical spaces, and smart objects [11], and by the capability to generate a wide amount of digital media contents and sensory effects that stimulate all senses. Multi-sensory

smart spaces offer opportunities for a countless variety of interaction-intensive and content-intensive user experiences that make this type of ISSs appropriate for many domains such as education, training, tourism, entertainment, or rehabilitation. The conceptual model discussed in this paper provides primitives to describe ISSs in the whole range of possible types.

\section{Modeling Interactive Smart Spaces}

The main abstractions of our conceptual approach are depicted in the metamodel shown in Figure 1. Abstractions are organized in two main sub-models: the Structural Model and the Interactive Behaviour Model. The Structural Model supports the representation of the human and technological "actors", their interaction capability, i.e., which actions they can perform and perceive ("sense") and which perceivable effects they can generate ("actuate"), as well as the digital contents that are involved in the user experience. The Interactive Behavior Model supports the representation of the interactive behaviour of all actors and how cross-interactions are orchestrated for the users to perform tasks and activities at different levels of complexity.

\subsection{Structural Model}

The Structural Model is built around the notion of Actors (both Human and Technological), that are the building blocks of any ISS as their properties and their cross-interactions enact the ISS interactive experience. Stimuli and Digital Resources then support the execution of the interactive experience. 


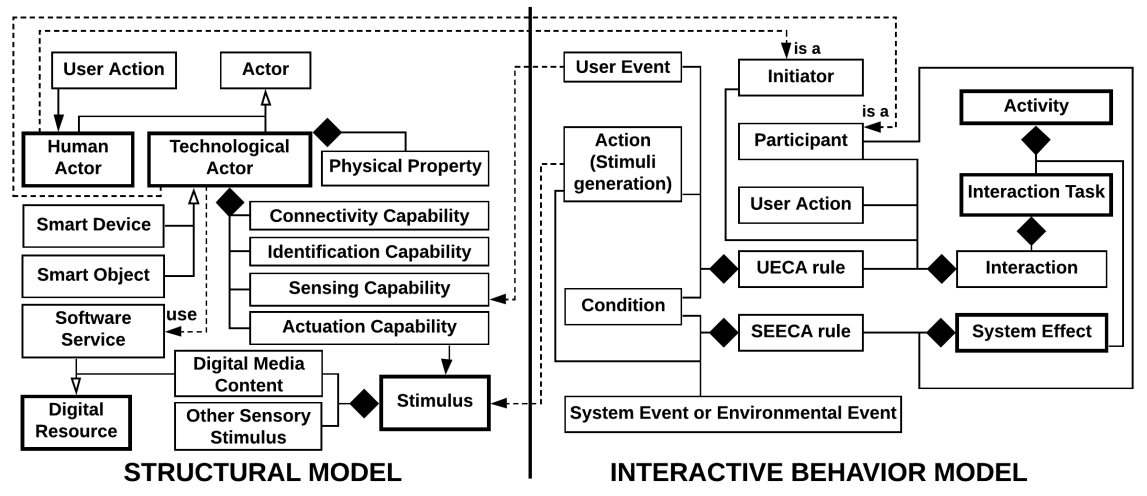

Fig. 1. Metamodel. Larger borders denote key elements.

Human Actor. Human Actors (i.e., the users), are described by their interaction capability, i.e., the User Actions (Actions for short) they are expected to perform while interacting with Technological Actors. Considering the user as an actor perceiving stimuli and acting on the surrounding space distinguishes an ISS from other cyber-physical systems that mainly consists of networks of physical and computational components.

Technological Actor. Technological Actors are characterized by a mix of digital and physical features. They make use of IoT equipment that enables one or more of the following capabilities: sensing (detecting signals generated by other Actors), actuation (generating data, content, or human-perceivable stimuli of any nature), connectivity (exchanging signals with other Technological Actors or systems), and identification (being identifiable in a unique way by other systems, e.g., by means of RFID tags). Technological Actors can also be characterized by physical properties, e.g., the material they are built of, the shape, or the physical affordance, if these "product design" features are relevant for the interactive experience. We envision two types of Technological Actors: smart devices and smart objects. Smart devices are self-standing (often off-the-shelf) equipment. Examples of smart devices with sensing capability only are luminosity sensors. Examples of smart devices with actuation capability only are smart lights, sound players, digital displays, or projectors. A tablet that the user brings around, "senses" the user's current position, and reacts by displaying related content, has both sensing and actuation capability. Smart objects are everyday life objects (e.g., a toy or a piece of furniture) or purposely-crafted physical items which are augmented with capabilities of sensing, actuation, connectivity, or identification. Compared with smart devices, modeling physical properties is more relevant for this class of Actors, as the interaction with them is typically tangible and involves some form of manipulation consistently with the object's affordance. Given the variety of technologies associated to ISSs, it is useful to describe Technological Actors at two levels. At a high level, Technological Actors are represented as mere "placeholders" of interaction capabilities, and "contain- 
ers" of one or more technological components that expose sensing, actuation, connectivity, or identification capabilities. Actuation capability is described in terms of stimuli, either elementary or complex.

Stimulus. A stimulus in our model is any phenomenon that can be generated by a digital device as "actuation" and can be perceived by user's senses: touch, sight, hearing, and smell. Stimuli can be mono- or multi-sensory, depending if they involve one or more senses. Images, animations, and lights are examples of visual stimuli. Sound and music are example of hearing stimuli. Example of multisensory stimuli are videos with aural content, bubbles generated by a bubble machine (which can be touched and seen) and the vibration of a smart toy (which might involve touch and sight).

Digital Resource. Digital Resources are digital-only, immaterial components that, depending on the type of ISS, contribute at different degrees to the user experience or are needed for computational purposes. Digital resources are of two types: i) Digital media content: in ISSs, many experiences are contentintensive (see Section 3) and content consumption is a large part of the user experience; mono- or multi-media content is generated by Technological Actors to give feedback during interaction, to create immersivity effects in the environment, to promote engagement, or to prompt the user during specific activities; ii) software services: these are software components exposing data and operations needed for computational purposes, and appear in the conceptual representation of the ISSs as placeholders to encapsulate technical features enabling interaction.

\subsection{Interactive Behaviour Model}

The Interactive Behavior Model provides the primitives to represent how the user experience unfolds along the time and how users interact with Technological Actors to reach their goals during the experience. It comprises two sub-models, at two levels of abstraction: the Activity Interaction Model and the Expanded Activity Interaction Model. Both models borrow some concepts and notations from the choreographic process model of BPMN [7]. BPMN is a well-known modeling language; it supports the specification of business processes and of collaborations among different stakeholders by means of a notation similar to UML Activity Diagrams. We re-interpret BPMN through the lens of human-ISS interaction, and extend/adapt some BPMN primitives to account for the specific modeling requirements of the human activities in ISSs, which are characterized by the "collaboration" between Human and Technological Actors.

Activity Interaction Model. This sub-model supports the high-level representation of the logic of the human activities in the ISS, and provides three abstractions: Activity, Interaction Task, and System Effect.

An Activity is a human "process" taking place within the ISS. It is represented by conventional flow diagrams composed by Interaction Tasks, System Effects, and control structures.

Interaction Tasks are the tasks performed by the user to reach the goal of the process. An Interaction Task encapsulates the Interactions, i.e., the user's actions with Technological Actors, needed to complete the task. At a high level, 
the representation of an Interaction Task declares what the users are expected to achieve (e.g., "select an item") but not how they achieve it, and with whom, omitting the specification of the specific Interactions and the associated Technological Actors. These latter aspects are represented in the Expanded Activity Interaction Model.

A System Effect is a set of perceivable, possible combined, stimuli generated automatically in response to System Events or Environmental Events, i.e., events that are outside of direct users' control. Such stimuli might have various goals, for example: to provide feedback when an Interaction Task is completed; to prompt the user to perform an Interaction Task; to increase engagement and serendipity at some point of time during the experience in the ISS; to adapt the physical space to weather condition. At high level, stimuli specifications are omitted in the representation. A System Effect declares what it is expected to achieve but not how, the latter being described in the Expanded Activity Interaction Model.

Expanded Activity Interaction Model. The Expanded Activity Interaction Model refines activity specifications by exposing the key features of Interaction Tasks and System Effects, zooming into the details of the specific User Actions performed within each Interaction Task and their direct effects (stimuli), and about the perceivable phenomena that take places place in the environment automatically during the Activity.

Each Interaction Task is described as a flow of Interactions. An Interaction is described by: 1) the Human Actor(s) performing the Interaction, hereinafter referred to as Initiator(s); 2) the User Action performed by the Initiator(s) according to the available interaction capability (as described in the Structural Model); 3) the Actors - either Human or Technological - participating in the Interaction, hereinafter referred to as Participants; 4) a set of UECA Rules, which relate User Actions to their direct effects, i.e., stimuli. UECA rules account for the intrinsically event-driven logic of human-technology interaction, and their specification is guided by the sensing and actuation capabilities of the participant Technological Actors (described in the Structural Model). UECA rules have an ECA (Event-Condition-Action) rule format where the Event is a "User Event" associated to the detection of the "User Action" by the Participant Technological Actor(s); the "Condition" predicates on the User Event; the "Action" describes the stimuli actuated by the Participant Technological Actor(s) if the Condition is satisfied.

Depending on the available technology, different Interactions can be included within the same Interaction Task to specify multiple ways to accomplish the task, using different interaction paradigms (e.g., based on movements, gestures, object manipulation, or voice). In this way, the model enables to represent multimodal interaction, which is one of the most relevant peculiarities of ISSs, particularly to support different user profiles and account for different user skills.

In the Expanded Behavioral Model, a System Effect is described by: Participants - Technological Actors holding the actuation capability needed to generate the required stimuli; a set of $S E E C A$ rules, i.e., ECA rules that define the System or Environmental Events that, under given conditions, trigger such stimuli. 


\section{Case Study: Magic Room}

We exemplify the concepts introduced in the previous sections by modeling the salient interaction features of the Magic Room. This is a sophisticated multisensory smart space for children's play, learning, and rehabilitation that we developed in the context of a national project and installed at two local schools and two therapeutic centers in Italy [13-16]. Children's experiences in the Magic Room have various goals: i) promoting well-being and relaxation; ii) providing controlled stimulation for the vestibular, proprioceptive and tactile sensory systems to improve "sensory integration" capability; iii) developing children's cognitive skills through learning-by-doing and embodied learning approaches. The design process of the Magic Room was participatory and iterative, and unfolded for a period of approximately four months, involving ten primary school teachers, three special educators, five therapists (psychologists, experts in neurodevelopmental disorders), and our technical team (two interaction designers and three computer engineers). Our model was used as a common language to communicate and externalize ideas during brainstorming, to share and discuss design proposals and alternatives at different levels of abstraction and refinement, and to integrate the contributions from all members of the heterogeneous project team. We organized three initial workshops devoted to: i) present to educators and therapists the technology we integrate; ii) explain the key concepts of our model and rephrase the description of technology in terms of the main Technological Actors and their interactive capabilities; iii) identify children's profiles and needs, and elicit caregivers' goals. Then educators and therapists focused on the definition of children's activities, working autonomously and in team, and progressively reporting the results to the technical team for discussion. Caregivers mainly used the Interactive Behavioral Model, in an informal way, drafting boxes (for Interaction Tasks and System Effects) and arrows (to indicate the temporal order among them) in a blackboard, identifying Initiators and Participants, and writing inside the boxes the textual descriptions of what they would like the users to do during each Interaction Task, and the generated stimuli. In parallel, they also identified some physical materials and objects useful for the various activities, and the interactive capability that would expect from them, paving the ground for the definitions of the Structural Model, finally mapping these Technological Actors to Activities as Participants in the various Interaction Tasks.

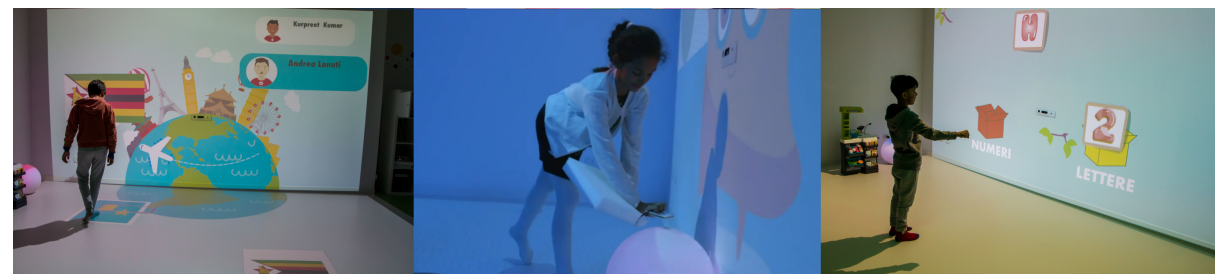

Fig. 2. Children playing in the Magic Room; in the centre: Interaction with the Smart Sphere 


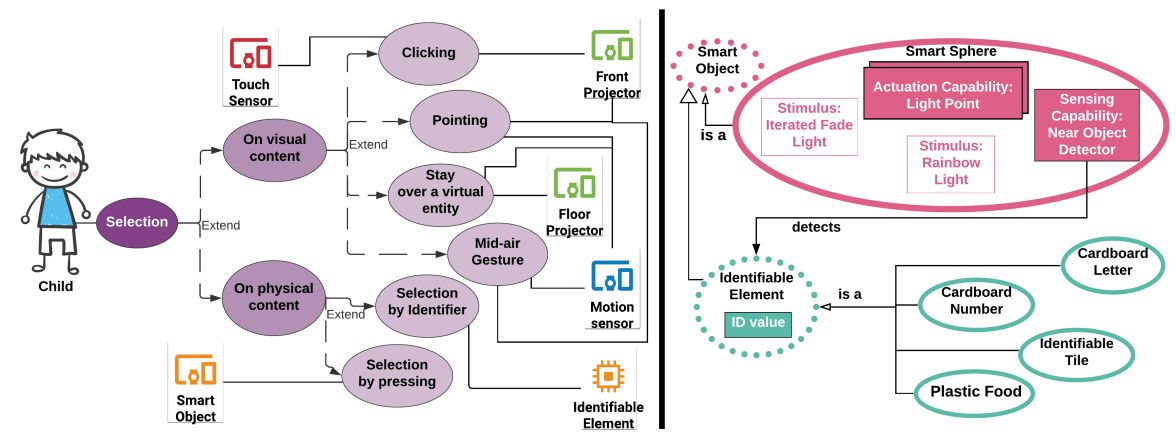

Fig. 3. Excerpts from the Structural Model of the Magic Room: the specification of a User Action, Selection, by the human actor Child (left). The high-level specification of the Technological Actor Smart Sphere (right).

The technical team elaborated these drafts and translated them into more precise specifications, using the full gamut of primitives of our model. Designers and engineers specified the Structural Model at high level and low level respectively. Engineers were also responsible of the full specifications of the Behavioral Model (refining the graphs for Interaction Task flows, System Effects, and associated rules). When needed, we reshaped some design solutions suggested by domain experts in light of the constraints of the technology, and returned back to them to discuss and validate the updated proposals. For lack of space, in the rest of the section we present only few examples of the conceptual model of the Magic Room, extracted from the specification of one activity - Battleship, a smart space version of classic battle-ship board game.

\subsection{Structural Model}

Actors and Digital Resources: High Level Model. The Human Actors in the Magic Room are Child and Caregiver. The Technological Actors are: front and floor projectors, Kinect body-motion sensor, RFID cards and readers attachable to various materials, portable and ambient smart lights, sound players, aroma and soap bubbles emitters, a tablet offering an app for caregiver to control the execution flow of Activities and Interaction Tasks. There are also several Smart Objects: Smart Toys (embedding motion and pressure sensors and light or sound actuators), Paper- or plastic-based Identifiable Objects (RFID tagged items), and a Smart Sphere (described in Figure 3 - right side). A Smart Sphere embeds a sensor (a Near Object Detector) able to generate an object identifier when it detects the proximity of an Identifiable Element). The Smart Sphere also includes a number of light actuators ("Light Points"). These can generate composite light effects such as Rainbow Light and Iterated Fade Light, represented on the right side of Figure 3.

The Magic Room also features a large number of Digital Media Content such as videos, animations, images, sounds, and music. 


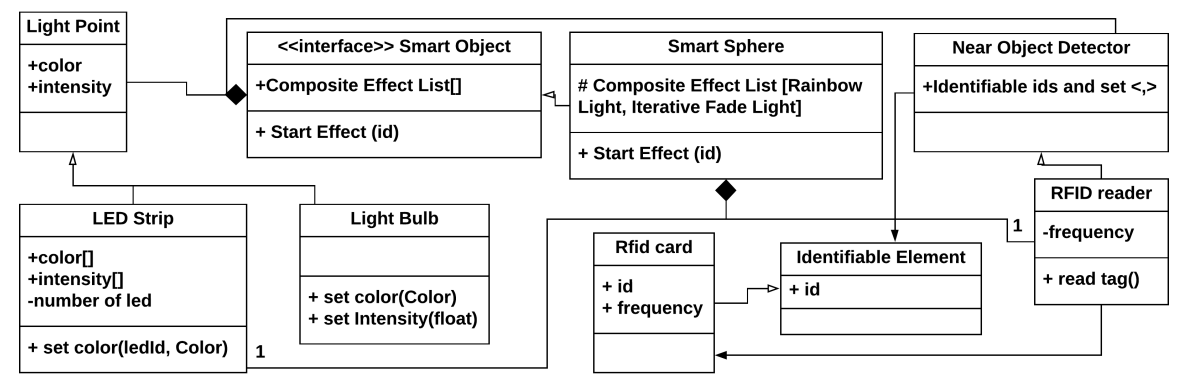

Fig. 4. Excerpts from the Low-Level Structural Model: the Smart Sphere.

Children interact with projections and smart objects, while Caregivers operate on the tablet.

Technological Actors: Low-Level Model. The UML class diagram in Figure 4 represents the Smart Sphere at a lower level, in terms of its attributes and functions. The Smart Sphere is a Smart Object that exposes two composite light stimuli (Rainbow and Fade) and a method to generate them. Its Light Points use LED strips, with associated attributes and methods controlling light effects. The Smart Sphere also uses a Near Object Detector to sense the proximity of RFID tags embedded in Identifiable Elements (RFID card) and to read its unique ID.

User Actions. Figure 3 illustrates an excerpt of Structural Model concerning the User Action Selection associated to the Human Actor "Child". The User Action is specified first by describing what can be selected, either visual or physical content, and then how the selection is performed through some Technological Actors. For example, the Child can "select" a visual content by executing a midair gesture (sensed by the motion sensor) in front of Digital Media Contents projected by the Wall Projector. The Child could stay over a virtual entity projected on the floor by the Floor Projector; s/he can also select by pressing, when interacting with physical content represented by a Smart Object; or s/he can select by identifier, using an Identifiable Element, i.e., a Smart Object that has identification features (embedding RFID cards) and can be detected by proximity by a Smart Objects that have RFID sensing capability (e.g., the Smart Sphere).

\subsection{Interactive Behaviour Model}

Interactive Activity Model. Figure 5 exemplifies the behavioral specification for the Activity "Battleship". The flow diagram (left side of Figure 5) includes the Interaction Task "GetCellCoordinates" to select a cell in the battleship grid, and a number of System Effects that generate visual and auditory feedback to outline partial or full hit, water hit, or end of game.

The fragment of the Expanded Activity Interaction Model shown on the right side of Figure 5 presents the details of the Interaction Task "GetCellCoordinates". The diagram provides two alternative modalities to accomplish this 

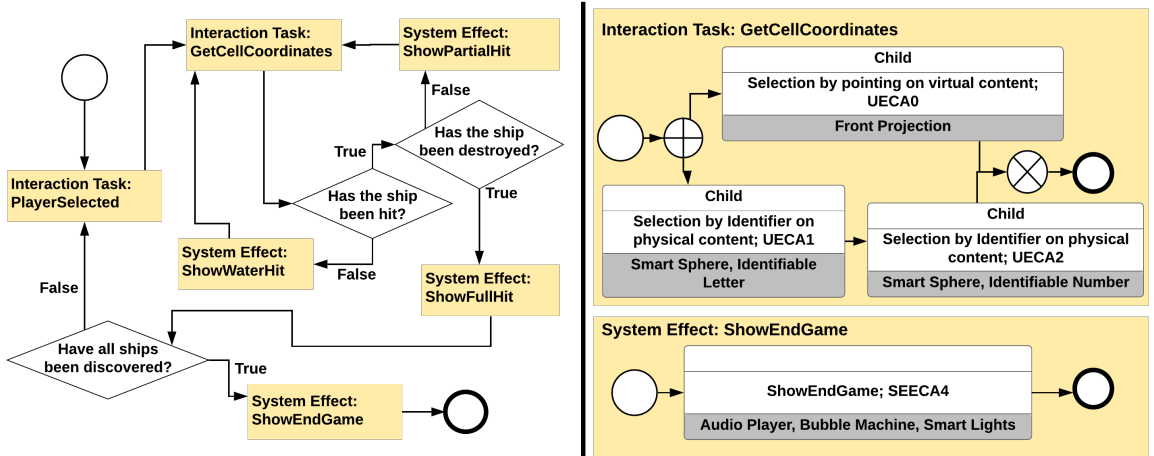

Fig. 5. Activity Interaction Model of Battleship (left) and the Expanded Activity Interaction Model for the Interaction Task enabling the cell coordinates' selection (right).

Interaction Task (mid air gestures and tangible interaction), each one associated to different Interactions: The user can either "point on" a virtual content (a cell of the Battle grid projected on the front screen), or can place - on top of the Smart Sphere - two Identifiable Cards for the cell coordinates, one with a number and one with a letter. Each box associated to an Interaction shows the Human Actor (Initiator) who executes the action(s) (upper area of the box) and the Participant Technological Actors (Smart Sphere and Identifiable Objects) involved in the Interaction (bottom area of the box). The middle area of the Interaction box includes the User Action (for example "Selection by Identifier on Physical content") and the UECA rules, for example "UECA 1", the specification of which (omitted in figure for lack of space) is: $<\boldsymbol{U S E \boldsymbol { E }}$ EVENT:Sensed (ID); CONDITION:Type(ID)= "Letter"; ACTION: Activate("Smart_Sphere", "rainbow_light")>. The rule means that if the Identifiable Object selected by the user and sensed by the Smart Sphere (Participant) is a letter (i.e., the first coordinate of the cell selection), then the Smart Sphere generates a multi-color, rainbow-like light stimulus.

At low level, a System Effect (see bottom of Figure 5 - right side) is represented by a box having empty top area (being the stimuli generated by System or Environmental Events without intentional human intervention) and SEECA rules, e.g., rule SEECA4, which defines the stimuli (music, lights, soap bubbles) generated at the end of the game when all the ships are sunk (System Event "game successfully completed").

\subsection{From Models to Software Architectures}

The abstractions presented in the previous sections guided the definition of a multi-layer architecture for the Magic Room (Figure 6) characterized by modularity, flexibility, and extensibility. The Activity specifications in the Interactive Behavioural Model and the Technology Actor specifications in the Structural 


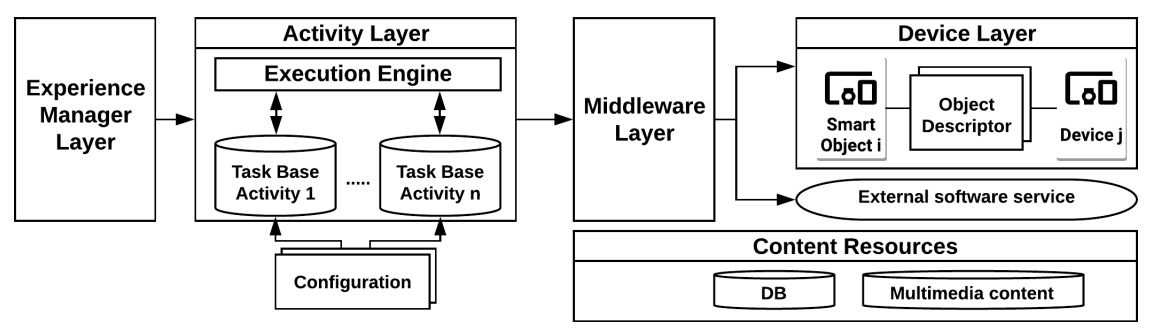

Fig. 6. Magic Room architecture

Model guide the definition of a JSON-based configuration file that the Execution Engine interprets as low-level rules governing the interactive capabilities of Technological Actors. During the execution of an Activity, the Execution Engine manages the transition between interactive tasks, and triggers the execution of the rules governing interactions, while the Experience Manager controls the flow of different activities. The Execution Engine leverages on the Content Resources component and on the Middleware, demanding to these modules the low-level execution of the activated rules. The Middleware manages the low-level functionality of each Technological Actor, as expressed in the Low-Level Structural Model, abstracting from the technology heterogeneity of the IoT products by means of adequate wrappers. The Middleware also supports the runtime discovery and instantiation of the different devices, depending on the interaction capabilities required by the Interaction Tasks in execution. To enhance a plug and play approach, devices expose their structural content and specific functions by means of Object Descriptors.

\section{Conclusions}

This paper has discussed some modeling requirements that characterize Interactive Smart Spaces (ISSs), pinpointing the importance of considering the human as the principal actor in this class of systems, and addressing the interaction capabilities as fundamental for empowering users in this spaces and enabling the accomplishment of complex tasks. Limited research in the IoT arena has taken this perspective so far. In addition, we have presented a novel conceptual model that enables designers of ISSs to focus on the salient human-centered aspects of IoT-enhanced user activities, and to organize the design along multiple dimensions and levels of abstractions. The proposed model deserves additional refinements, but also in its current form it has demonstrated its expressive power and its capability of supporting the design and conceptual specification of a very complex, content-intensive and interaction-intensive smart space, discussed as case study in the paper. Our model and our overall approach can pave the ground towards innovative methods for conceptual design in the IoT arena, and may also lead to the definition of more modular and standardized technological architectures for future highly interactive IoT systems. 
An interaction-centered modeling approach is also the first step towards the definition of novel solutions to support the appropriation process of interactive IoT technology by end users; adequate abstractions like the ones presented in this paper can provide a base of concepts upon which to create the building blocks for End-User Development methods and tools [10]. This issue is particularly important in contexts - like education and rehabilitation - where Interactive Smart Spaces could have a significant potential but there is a strong need of personalization of the user experiences. Since it is not possible to predict at design time all the possible relevant configurations of activities as built-in components of an ISS, users would benefit from the possibility of eventually defining their interactive experiences or configuration parameters by themselves [1], without any knowledge about the underlying implementation. This view raises new research challenges, addressing the way the interaction capabilities of ISS should be modelled through metaphors and design patterns that make sense to the users, and would enable them to customize or even create from scratch their own interactive smart experiences, starting from flexible activity skeletons and a repertoire of interactive capabilities that they can manage and organize.

\section{References}

1. Ardito, C., Buono, P., Desolda, G., Matera, M.: From smart objects to smart experiences: An end-user development approach. IJHCS 114, 51-68 (2018)

2. Arruda, M.F., Bulcão-Neto, R.F.: Toward a lightweight ontology for privacy protection in IoT. In: Proceedings of SAC' 19. pp. 880-888. ACM (2019)

3. Atzori, L., Iera, A., Morabito, G.: The internet of things: A survey. Computer networks 54(15), 2787-2805 (2010)

4. Bassi, A., Bauer, M., Fiedler, M., van Kranenburg, R., Lange, S., Meissner, S., Kramp, T.: Enabling things to talk. Springer Nature (2013)

5. Bermudez-Edo, M., Elsaleh, T., Barnaghi, P., Taylor, K.: IoT-lite: A lightweight semantic model for the internet of things and its use with dynamic semantics. Personal Ubiquitous Comput. 21(3), 475-487 (2017-06)

6. Conti, M., Das, S.K., Bisdikian, C., Kumar, M., Ni, L.M., Passarella, A., Roussos, G., Tröster, G., Tsudik, G., Zambonelli, F.: Looking ahead in pervasive computing: Challenges and opportunities in the era of cyber-physical convergence. Pervasive Mob. Comput. 8(1), 2-21 (2012)

7. Cortes-Cornax, M., Dupuy-Chessa, S., Rieu, D., Dumas, M.: Evaluating choreographies in bpmn 2.0 using an extended quality framework. In: International Workshop on Business Process Modeling Notation. pp. 103-117. Springer (2011)

8. Costa, B., Pires, P.F., Delicato, F.C.: Modeling SOA-based IoT applications with SoaML4iot. pp. 496-501. IEEE (2019-04)

9. Delprino, F., Piva, C., Tommasi, G., Gelsomini, M., Izzo, N., Matera, M.: Abbot: a smart toy motivating children to become outdoor explorers. In: Proc. of AVI'18. pp. 1-9 (2018)

10. Desolda, G., Ardito, C., Matera, M.: Empowering end users to customize their smart environments: model, composition paradigms, and domain-specific tools. ACM Transactions on Computer-Human Interaction (TOCHI) 24(2), 1-52 (2017)

11. Foglia, L., Wilson, R.A.: Embodied cognition. WIREs Cognitive Science 4(3), 319$325(2013)$ 
12. Fortino, G., Russo, W., Savaglio, C., Shen, W., Zhou, M.: Agent-oriented cooperative smart objects: From IoT system design to implementation. IEEE Transactions on Systems, Man, and Cybernetics: Systems 48(11), 1939-1956 (2018-11)

13. Garzotto, F., Beccaluva, E., Gianotti, M., Riccardi, F.: Interactive multisensory environments for primary school children. In: Proc. of CHI '20. p. 1-12. CHI '20, Association for Computing Machinery, New York, NY, USA (2020)

14. Garzotto, F., Gelsomini, M.: Magic room: A smart space for children with neurodevelopmental disorder. IEEE Pervasive Computing 17(1), 38-48 (2018)

15. Gelsomini, M., Leonardi, G., Garzotto, F.: Embodied learning in immersive smart spaces. In: Proc. of CHI '20. pp. 1-14 (2020)

16. Gelsomini, M., et al.: Magika, a multisensory environment for play, education and inclusion. In: Extended Abstracts of the 2019 CHI Conference on Human Factors in Computing Systems. pp. 1-6 (2019)

17. Gračanin, D., Handosa, M., Elmongui, H.G., Matković, K.: An approach to user interactions with iot-enabled spaces. In: Proc. of ConTEL'17. pp. 139-146. IEEE (2017)

18. Haller, S., Serbanati, A., Bauer, M., Carrez, F.: A domain model for the internet of things. In: 2013 Proc. of Green Computing and Communications and Internet of Things and Cyber, Physical and Social Computing, 2013. pp. 411-417 (2013-08)

19. Jahed, K., Dingel, J.: Enabling model-driven software development tools for the internet of things. In: Proc. of MiSE '19. pp. 93-99. IEEE Press (2019)

20. Kortuem, G., Kawsar, F.: Market-based user innovation in the internet of things. In: 2010 Internet of Things (IOT). pp. 1-8. IEEE (2010)

21. Maheswaran, M., Wen, J., Gowing, A.: Design of a context aware object model for smart spaces, things, and people. In: Proc. of IEEE ICC'15. pp. 710-715 (2015-06)

22. Markopoulos, P., Marijnissen, P.: Uml as a representation for interaction designs. In: Proceedings of OZCHI 2000. pp. 240-249 (2000)

23. Ning, D., Wang, Y., Guo, J.: A data oriented analysis and design method for smart complex software systems of IoT. In: Proc. of ISSI'18. pp. 1-6 (2018-09)

24. Petrelli, D., Lechner, M.: The mesch project-material encounters with digital cultural heritage: Reusing existing digital resources in the creation of novel forms of visitor's experiences. Proc. CIDOC'14 (2014)

25. Sahinel, D., Akpolat, C., Görür, O.C., Sivrikaya, F.: Integration of human actors in IoT and CPS landscape. In: Proc. of WF-IoT. pp. 485-490 (2019-04)

26. Skarmeta, A., Hernández-Ramos, J.L., Bernabe, J.B.: A required security and privacy framework for smart objects. In: 2015 ITU Kaleidoscope: Trust in the Information Society (K-2015). pp. 1-7 (2015-12)

27. Weiser, M.: The computer for the 21 st century. Sc. American 265(3), 94-105 (1991)

28. Zeng, J., Yang, L.T., Ma, J.: A system-level modeling and design for cyber-physicalsocial systems. ACM Trans. Embed. Comput. Syst. 15(2), 35:1-35:26 (2016-05)

29. Zhao, Y., Pour, F.F., Golestan, S., Stroulia, E.: BIMSim3d: Multi-agent human activity simulation in indoor spaces. In: Proc. of the Int. Workshop on Software Engineering for Smart Cyber-Physical Systems. pp. 18-24. IEEE Press (2019) 